\title{
THE DYNAMIC EPISTEMIC LOGIC FOR ACTUAL KNOWLEDGE
}

\begin{abstract}
The dynamic epistemic logic for actual knowledge models the phenomenon of actual knowledge change when new information is received. In contrast to the systems of dynamic epistemic logic which have been discussed in the past literature, our system is not burdened with the problem of logical omniscience, that is, an idealized assumption that the agent explicitly knows all classical tautologies and all logical consequences of his or her knowledge. We provide a sound and complete axiomatization for this logic.
\end{abstract}

Keywords: Dynamic epistemic logic, logic of public announcements, knowledge representation, problem of logical omniscience, actual knowledge, epistemic change, multi-agent systems.

\section{Introduction}

During the mid-twentieth century, the attention of many logicians and philosophers focused on epistemic modalities, and the first systems of epistemic logic were developed, such as those of Jerzy Łoś [10] and Arthur Pap [11]. An interest in these systems was then heightened when Jaakko Hintikka [5] applied the concept of possible worlds semantics to epistemic operators. Originally, possible worlds semantics was formulated by Saul Kripke [6, 7] for the logic of necessity and possibility. The semantics developed by Kripke has since been adapted to epistemic logic, prompting the development of modal epistemic logics. Modal epistemic logics have become not only helpful tools for the formalization of certain intuitions 
connected with the concepts of knowledge, belief and information; but have also become a subject of interest to scientists in fields such as game theory, computer science, cognitive science, decision theory, artificial intelligence (AI) and cryptology.

The increase of interest in epistemic logic among representatives of other scientific disciplines has lead to new goals for the application of the logic of knowledge and beliefs. Epistemic logics started to be perceived as formal systems whose aim is to capture the phenomenon of epistemic change as a result of the flow of information between various agents. But standard modal epistemic logics have a clearly static character: they model the agent's information state at a given time, but do not enable us to express how this state can change when new information is received. Thus, the need to develop formal systems that allow for capturing the phenomenon of epistemic change led to a dynamic turnover in epistemic logic.

Dynamic epistemic logics are not free of the shortcomings typical of standard epistemic logics. Both make use of possible worlds semantics, and inherit its drawbacks. This concerns logical omniscience, i.e. the controversial assumption underlying epistemic logics built on the basis of possible worlds semantics, according to which the agent knows all classical (propositional) tautologies, and all logical consequences of his or her knowledge.

So far, at least a dozen different proposals have been made to solve the problem of logical omniscience ${ }^{1}$. Many authors have recognized that this problem illustrates the fact that epistemic logics do not model the actual knowledge of agents (explicit knowledge), but only potential knowledge (implicit knowledge), for which the discussed assumptions are not problematic (Levesque [8], Fagin, Halpern [2], van Benthem [1]). If standard epistemic logic models potential knowledge, it is still an open question how to develop a system that enables us to model actual knowledge. Ronald Fagin and Joseph Y. Halpern [2] have constructed such a logic for actual knowledge of non-omniscient agents. The main aim of this article is to develop a dynamic epistemic logic that is built on the ideas formulated by Fagin and Halpern. We present the axiomatization for such a system, we propose a semantics for it and finally we prove the soundness and completeness theorem.

\footnotetext{
${ }^{1}$ The most important of these proposals are discussed in detail by Fagin et al. [3] and $\operatorname{Sim}[15]$.
} 


\section{Modal epistemic logics}

The formal language of modal epistemic logics $\mathcal{L}_{M E L}$, is the common language for a wide class of logics.

Definition 1. Let $\operatorname{Var}$ denote the set of sentential variables and let $A g$ denote the set of agents. The language of modal epistemic logics $\mathcal{L}_{M E L}$ is defined inductively as follows:

$$
\varphi::=p|\neg \varphi| \varphi \rightarrow \varphi \mid K_{i} \varphi,
$$

where $p \in \operatorname{Var}$ and $i \in A g$. The set of all $\mathcal{L}_{M E L}$ formulas is denoted by $\Gamma_{\mathcal{L}_{M E L}}$

$\mathcal{L}_{M E L}$ is an extension of the language of propositional logic with epistemic operators $K_{i}$ for every agent $i \in A g$. The intended interpretation of $K_{i} \varphi$ is "agent $i$ knows that $\varphi$ ". In the case where we are only dealing with one agent, we can omit the index. Other classical logical constants can be defined in the standard way.

The semantics of modal epistemic logics are constructed on the basis of the semantics that Kripke [6, 7] proposed for the logic of possibility and necessity.

Definition 2. An epistemic model is a structure $\mathcal{M}=\left(W,\left\{R_{i}: i \in A g\right\}, v\right)$, where

- $W \neq \emptyset$ is a set of epistemic states,

- $A g \neq \emptyset$ is a set of agents,

- $R_{i} \subseteq W \times W$ is an epistemic accessibility relation for any $i \in A g$,

- $v: \operatorname{Var} \mapsto \mathcal{P}(W)$ is a valuation function which to every $p \in \operatorname{Var}$ assigns the set of epistemic states in which $p$ is true.

Definition 3. Let $\mathcal{M}=\left(W,\left\{R_{i}: i \in A g\right\}, v\right)$ be an epistemic model. The satisfiaction relation $\models$ is defined inductively in the following way:

$$
\begin{array}{lll}
\mathcal{M}, s \models p & \text { iff } & s \in v(p), \\
\mathcal{M}, s \models \neg \varphi & \text { iff } & \mathcal{M}, s \not \varphi, \\
\mathcal{M}, s \models \varphi \rightarrow \psi & \text { iff } & \text { if } \mathcal{M}, s \models \varphi, \text { then } \mathcal{M}, s=\psi, \\
\mathcal{M}, s \models K_{i} \varphi & \text { iff } & \text { for all } t \in W: \text { if }(s, t) \in R_{i}, \text { then } \mathcal{M}, t=\varphi,
\end{array}
$$

where $p \in \operatorname{Var}, \varphi, \psi \in \Gamma_{\mathcal{L}_{M E L}}$, and $i \in A g$.

Definition 4. A formula $\varphi \in \Gamma_{\mathcal{L}_{M E L}}$ is true in an epistemic model $\mathcal{M}=$ $\left(W,\left\{R_{i}: i \in A g\right\}, v\right)$ whenever for any $s \in W, \mathcal{M}, s \models \varphi$. We denote this 
by $\mathcal{M} \models \varphi$. A formula $\varphi \in \Gamma_{\mathcal{L}_{M E L}}$ is valid whenever for any epistemic model $\mathcal{M}, \mathcal{M} \models \varphi$. We denote this by $\models \varphi$.

Let us start from the minimal modal epistemic logic which is denoted as $\mathbf{K}$.

Definition 5. A proof system for the logic $\mathbf{K}$ is given by the following axiom schemes and inference rules:

all instantiations of propositional tautologies

$K_{i}(\varphi \rightarrow \psi) \rightarrow\left(K_{i} \varphi \rightarrow K_{i} \psi\right)$

The inference rules:

from $\varphi \rightarrow \psi$ and $\varphi$ infer $\psi$

modus ponens

from $\vdash \varphi$ infer $\vdash K_{i} \varphi$

Gödel's rule for $K_{i}$

A formula is a $\mathbf{K}$-theorem if it belongs to the least set of formulas that contain all the axioms, and is closed under the inference rules. If $\varphi$ is a K-theorem, we write $\vdash_{\mathrm{K}} \varphi$.

The axiom $\mathrm{K}$ states that the knowledge operator is closed under implication. This axiom is accepted in every system of modal epistemic logic. A list of other familiar epistemic axioms is provided in Table 1.

Table 1. Axioms for knowledge

\begin{tabular}{|c|l|}
\hline Name & Axiom \\
\hline D & $K_{i} \varphi \rightarrow \neg K_{i} \neg \varphi$ \\
T & $K_{i} \varphi \rightarrow \varphi$ \\
4 & $K_{i} \varphi \rightarrow K_{i} K_{i} \varphi$ \\
5 & $\neg K_{i} \varphi \rightarrow K_{i} \neg K_{i} \varphi$ \\
\hline
\end{tabular}

Proof systems for the epistemic logics stronger than $\mathbf{K}$ are obtained by adding other axioms to the system. Table 2 lists the most important of these logics. It should be emphasized that the S5 logic is generally considered to be the standard epistemic logic. We get a proof system for S5 by expanding the proof system for $\mathbf{K}$ with the axioms $\mathbf{T}, 4$, and 5 . 
Table 2. Some basic epistemic systems

\begin{tabular}{|c|c|l|}
\hline Name & Axioms & Properties of $R_{i}$ \\
\hline $\mathbf{K D}$ & $\mathbf{K} \cup\{\mathrm{D}\}$ & serial \\
$\mathbf{T}$ & $\mathbf{K} \cup\{\mathrm{T}\}$ & reflexive \\
$\mathbf{S} 4$ & $\mathbf{T} \cup\{4\}$ & reflexive, transitive \\
$\mathbf{S 5}$ & $\mathbf{S} 4 \cup\{5\}$ & reflexive, symmetric, transitive \\
$\mathbf{K D} 4$ & $\mathbf{K D} \cup\{4\}$ & serial, transitive \\
$\mathbf{K D} 45$ & $\mathbf{K D} 4 \cup\{5\}$ & serial, transitive, euclidean \\
\hline
\end{tabular}

The standard modal epistemic logics are sound and complete with respect to classes of models whose accessibility relations have the properties expressed in the first-order language, and specified in Table 2.

THEOREM 1. Logic S5 is sound and complete with respect to the class of all equivalence epistemic models, i.e. any formula $\varphi \in \Gamma_{\mathcal{L}_{M E L}}$ is an $\mathbf{S 5 -}$ theorem iff $\varphi$ is true in all epistemic models, where the accessibility relations are equivalence relations.

The corresponding theorems for other epistemic logics can be formulated in the same way based on the content of Table 2. The logic $\mathbf{K}$ is sound and complete with respect to the class of epistemic models with arbitrary accessibility relations.

Epistemic logics based on possible worlds semantics suffer from the problem of logical omniscience. The problem of logical omniscience is connected to two rules of inference. This is the Gödel rule according to which the agent knows all theorems of a given epistemic logic, including all classical propositional tautologies, and the monotonicity rule which may be formulated in the following way:

if $\vdash \varphi \rightarrow \psi$, then $\vdash K_{i} \varphi \rightarrow K_{i} \psi$.

This rule is a consequence of the application of the Gödel rule and the rule of modus ponens to the axiom K. It implies that the agent knows all logical consequences of his or her knowledge. In some applications of epistemic logics, e.g. in epistemology and game theory, representing the knowledge of agents with unlimited deductive abilities may be accepted as a justified idealization. But in the case of representing knowledge of real cognitive agents, these unrealistic assumptions are undesirable. 


\section{The Fagin and Halpern logic}

In order to solve the problem of logical omniscience, Fagin and Halpern [2] propose to add a new epistemic operator to the standard, modal epistemic logic: an awareness operator. The set of formulas of such an extended language is characterized in accordance with the following definition.

Definition 6. Let $\operatorname{Var}$ be the set of sentential variables, and let $A g$ be the set of agents. The language of the modal epistemic logics with awareness operator $\mathcal{L}_{M E L-A}$ is defined inductively as follows:

$$
\varphi::=p|\neg \varphi| \varphi \rightarrow \varphi\left|K_{i} \varphi\right| A_{i} \varphi
$$

where $p \in \operatorname{Var}$ and $i \in A g$. The set of all $\mathcal{L}_{M E L-A}$ formulas is denoted by $\Gamma_{\mathcal{L}_{M E L-A}}$.

The intended interpretation of $A_{i} \varphi$ is "the agent $i$ is aware that $\varphi$ " or "the agent $i$ is informed that $\varphi^{\prime 2}$. The $A_{i}$ operator can be applied to a formula independently of the $K_{i}$ operator.

Fagin and Halpern [2] note that if an agent knows something, then the agent cannot be completely unaware of it. For this reason, the authors introduce a distinction between potential and actual knowledge. The potential knowledge is modeled by the operator $K_{i}$ for any $i \in A g$, while we are concerned with the actual knowledge that $\varphi$, when $\varphi$ is a subject of potential knowledge and the agent is aware that $\varphi$. Thus, we have a new epistemic operator - the actual knowledge operator defined in the language $\mathcal{L}_{M E L-A}$ for any formula $\varphi$ and any agent $i \in A g$ as follows:

$E_{i} \varphi \stackrel{\text { def }}{=} A_{i} \varphi \wedge K_{i} \varphi$

The semantics for $\mathcal{L}_{M E L-A}$ is a modified version of the semantics for $\mathcal{L}_{M E L}$.

Definition 7. A model of $\mathcal{L}_{M E L-A}$ is the epistemic model of Definition 2 with the awareness function: $\mathcal{M}=\left(W,\left\{R_{i}: i \in A g\right\},\left\{\mathcal{A}_{i}: i \in A g\right\}, v\right)$, where $\mathcal{A}_{i}: W \mapsto \mathcal{P}\left(\Gamma_{\mathcal{L}_{M E L-A}}\right)$ is the awareness function for any $i \in A g$.

\footnotetext{
[14].

${ }^{2}$ The philosophical aspects of the awareness approach are discussed in detail by Sillari
} 
Definition 8. Let $\mathcal{M}=\left(W,\left\{R_{i}: i \in A g\right\},\left\{\mathcal{A}_{i}: i \in A g\right\}, v\right)$ be an epistemic model with the awareness function and let $i \in A g$. Then Definition 3 is extended with the following condition:

$\mathcal{M}, s \models A_{i} \varphi \quad$ iff $\quad \varphi \in \mathcal{A}_{i}(s)$.

Referring to this definition and the way the $E_{i}$ operator was defined for any $i \in A g$, we get the following condition for the actual knowledge operator:

$\mathcal{M}, s=E_{i} \varphi \quad$ iff $\quad \varphi \in \mathcal{A}_{i}(s)$ and for all $t \in W$ : if $(s, t) \in R_{i}$, then $\mathcal{M}, t \models \varphi$.

Let $\mathbf{L}$ denote the modal epistemic logic, while $\mathbf{L}-\mathbf{A}$ denotes its extension obtained by adding to the proof system of $\mathbf{L}$ all formulas which are instantiations of the following schema:

$E_{i} \varphi \leftrightarrow\left(A_{i} \varphi \wedge K_{i} \varphi\right)$.

Fagin and Halpern [2, p. 67] proved the soundness and completeness of KD45-A logic with respect to the above-mentioned semantics with the awareness function ${ }^{3}$. The soundness and completeness of S5-A can be proved in a completely analogous fashion.

THEOREM 2. Logic S5-A is sound and complete with respect to the class of all equivalence epistemic models with the awareness function, i.e. any

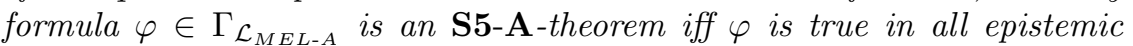
models with the awareness function, where the accessibility relations are equivalence relations.

It should be noted that the $E$ operator does not behave like a normal modal operator. In particular, the formula $E_{i}(p \vee \neg p)$ is not valid in epistemic logics with awareness, because agents may not realize that $p \vee \neg p$. There is also no equivalence between the formulas $E_{i}(p \wedge q)$ and $E_{i}(q \wedge p)$, because we may want to model the knowledge of an agent who does not have to see the equivalence relation between the formulas $p \wedge q$ and $q \wedge p$. The equivalence of Axiom 4 and 5 do not have to apply to the actual knowledge - although, of course, it is possible to add such axioms to the proof systems. Neither $\left(E_{i} \varphi \wedge A_{i} E_{i} \varphi\right) \rightarrow E_{i} E_{i} \varphi$, nor $\left(\neg E_{i} \varphi \wedge A_{i} \neg E_{i} \varphi\right) \rightarrow E_{i} \neg E_{i} \varphi$ has to be valid in Fagin and Halpern's logic. It should be emphasized, however,

\footnotetext{
${ }^{3}$ The authors use the concepts of knowledge and belief interchangeably. They work primarily on the logic KD45-A, because the acceptance of the $\mathrm{T}$ axiom for beliefs is not justified.
} 
that some properties of explicit knowledge are equivalent to properties of implicit knowledge. The schema for actual knowledge equivalent to the $\mathrm{K}$ axiom is $\left(E_{i} \varphi \wedge E_{i}(\varphi \rightarrow \psi) \wedge A_{i} \psi\right) \rightarrow E_{i} \psi$. The rule corresponding to the Gödel rule has now the following form: if $\vdash \varphi$, then $\vdash A_{i} \varphi \rightarrow E_{i} \varphi$.

Obviously, actual knowledge will have additional properties once we put some further restrictions on the awareness function. For example, the fact that the order of presentation of the conjuncts does not matter can be captured by the axiom $A_{i}(\varphi \wedge \psi) \leftrightarrow A_{i}(\psi \wedge \varphi)$, and in systems satisfying this restriction $E_{i}(\varphi \wedge \psi) \leftrightarrow E_{i}(\psi \wedge \varphi)$ is a valid formula. The fact that an agent is aware of a formula if and only if he is aware of its negation can be captured by the axiom $A_{i} \varphi \leftrightarrow A_{i} \neg \varphi$, and in systems satisfying this restriction $E_{i} \varphi \leftrightarrow E_{i} \neg \neg \varphi$ is valid.

\section{Dynamic epistemic logic for non-omniscient agents}

Our goal will be to construct a dynamic epistemic logic for actual knowledge of non-omniscient agents. Although similar motivations have been formulated by Rasmussen [13], the author developed his system only from an axiomatic point of view and has not provided a model theory for his logic.

In the case of the epistemic logics discussed so far, we have considered only static semantics, and logics of this kind do not allow for modeling the phenomenon of the agent's knowledge change when new information is received. Dynamic logics, starting from the logic of public announcements presented by Plaza [12] and Gerbrandy and Groeneveld [4], enable us to model the phenomenon of knowledge change.

The main idea associated with modeling updated knowledge is that whenever an agent receives new information, all epistemic states that are contradictory with this information are removed from an epistemic model that represents the agent's knowledge before the new information is received. The new information causes a transition from a certain initial epistemic model to its sub-model, that is, a model bounded by this new information. This is best illustrated by the so-called Muddy Children Puzzle, which comes from the book written by Littlewood (1953).

EXAMPLE 1. Three children come back home after playing in the garden. During the game, children could get mud on their foreheads. Each child 
sees the foreheads of the other two, however no child can see his or her own forehead. Father arranges the children in a row, and then says:

Father: At least one of you has a dirty forehead.

After this announcement he asks:

Father: If you know whether your forehead is dirty, then step forward

None of the children step forward. Father repeats himself a second time. Again nothing happens. Yet, when the father repeats himself for a third time, all of the remaining children step forward.

Let us assume that we have the following trio of children: $a, b, c$. We will use propositional variables $p_{a}, p_{b}, p_{c}$ to denote that relevant children have dirty foreheads. The graphical representation of the initial model of this situation is shown in Figure 1. The relation of accessibility in this model is reflexive, but the arrows symbolising this fact are left out from the figures in order to achieve better graphical clarity.

The sequence of epistemic interactions begins from the father's first announcement: "At least one of you has a dirty forehead", which means that $p_{a} \vee p_{b} \vee p_{c}$ holds. Let us note that $\mathcal{M}, 7 \not \models p_{a} \vee p_{b} \vee p_{c}$. Therefore world 7 is contradictory to the introduced information and should be eliminated. We leave it to the reader to analyze consequences of the remaining announcements and to solve the puzzle.

Definition 9. Let $\mathcal{M}=\left(W,\left\{R_{i}: i \in A g\right\},\left\{\mathcal{A}_{i}: i \in A g\right\}, v\right)$ be an epistemic model with the awareness function. The updated model by the new information $\varphi$ is defined as a tuple $\mathcal{M} \mid \varphi=\left(W^{\prime},\left\{R_{i}^{\prime}: i \in A g\right\},\left\{\mathcal{A}_{i}^{\prime}: i \in A g\right\}, v^{\prime}\right)$, where:

$$
\begin{aligned}
& W^{\prime}=\{s \in W: \mathcal{M}, s=\varphi\}, \\
& R_{i}^{\prime}=R_{i} \cap\left(W^{\prime} \times W^{\prime}\right), \text { for any } i \in A g, \\
& \mathcal{A}_{i}^{\prime}=\left.\mathcal{A}\right|_{W^{\prime}}, \text { for any } i \in A g, \\
& v^{\prime}(p)=v(p) \cap W^{\prime}, \text { for any } p \in \text { Var. }
\end{aligned}
$$

In other words, the model $\mathcal{M} \mid \varphi$ is the model $\mathcal{M}$ restricted to all those epistemic states where $\varphi$ holds.

To express that a sentence is true as a result of an announcement, we expand the language $\mathcal{L}_{M E L-A}$ by the dynamic operator $[\varphi] \psi$ for any formulas $\varphi, \psi \in \Gamma_{\mathcal{L}_{M E L-A}}$. 


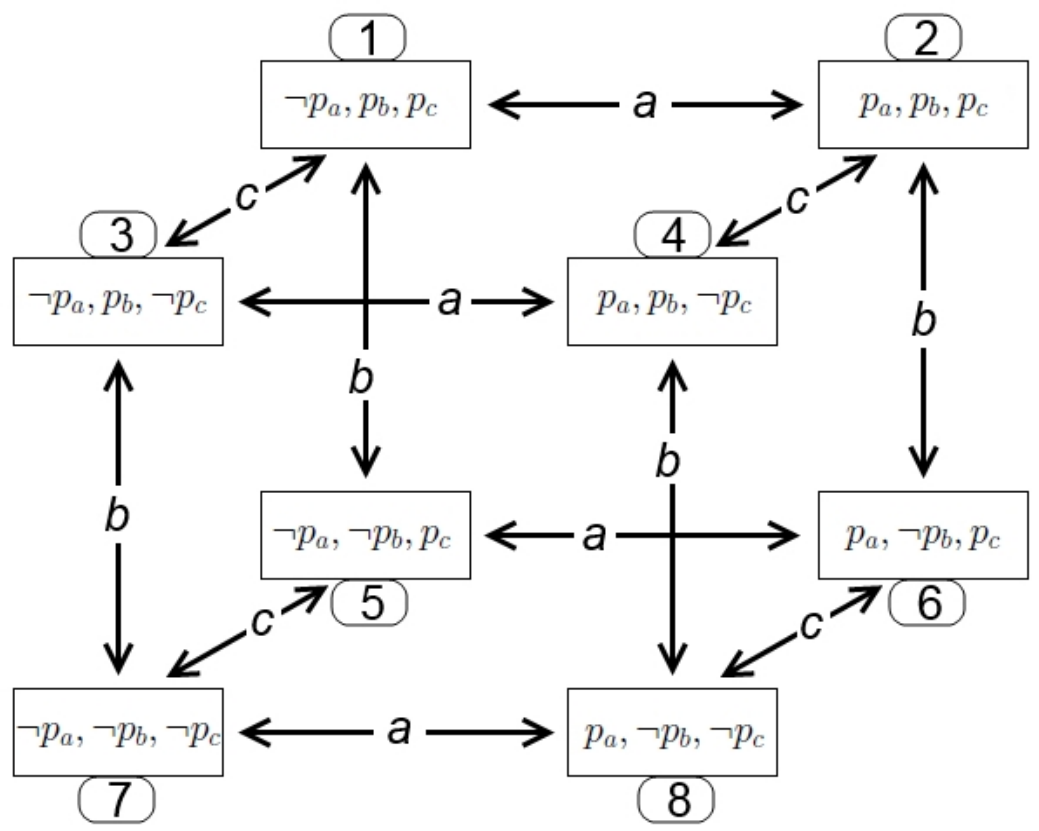

Fig. 1. Model $\mathcal{M}$ (initial model)

Definition 10. Let $\operatorname{Var}$ be the set of sentential variables and let $A g$ be the set of agents. The language of dynamic epistemic logic with the awareness operator $\mathcal{L}_{D E L-A}$ is defined inductively as follows:

$$
\varphi::=p|\neg \varphi| \varphi \rightarrow \varphi\left|K_{i} \varphi\right| A_{i} \varphi \mid[\varphi] \varphi,
$$

where $p \in \operatorname{Var}$ and $i \in A g$. The set of all $\mathcal{L}_{D E L-A}$ formulas is denoted by $\Gamma_{\mathcal{L}_{D E L-A}}$.

The narrow interpretation of the formula $[\varphi] \psi$ is "after a public announcement $\varphi$, it holds that $\psi$ ". However, the default interpretation of the dynamic operator is wider, and $[\varphi] \psi$ should be read as "after an epistemic update with $\varphi$, it holds that $\psi$ " or simply "after obtaining information $\varphi$, it holds that $\psi$ ". Thus, one may get the updated epistemic model not only as a result of public announcements but also as a result of other acts, such as 
observation or positive verification. Depending on the context, a formula $[\varphi] \psi$ can be interpreted, for example, as "after the observation that $\varphi$, it holds that $\psi$ " or "after publicly verifying the truth of $\varphi$, it holds that $\psi$ ".

Definition 11. Let $\mathcal{M}=\left(W,\left\{R_{i}: i \in A g\right\},\left\{\mathcal{A}_{i}: i \in A g\right\}, v\right)$ be an epistemic model with the awareness function and let $s \in W$. Then Definition 8 is extended with the following condition:

$\mathcal{M}, s \models[\varphi] \psi \quad$ iff $\quad$ if $\mathcal{M}, s \models \varphi$, then $\mathcal{M} \mid \varphi, s \models \psi$,

where $\mathcal{M} \mid \varphi$ is a model $\mathcal{M}$ restricted to all those epistemic states where $\varphi$ holds.

We propose the following proof system for the logic DEL-A, i.e. the dynamic epistemic logic for actual knowledge of non-omniscient agents.

Definition 12. A proof system for the logic DEL-A with the operators $K_{i}$ and $A_{i}$ for any $i \in A g$, and the dynamic operator [ ] is given by the following axioms and inference rules:

all instantiations of propositional tautologies

$K_{i}(\varphi \rightarrow \psi) \rightarrow\left(K_{i} \varphi \rightarrow K_{i} \psi\right)$

$K_{i} \varphi \rightarrow \varphi$

$K_{i} \varphi \rightarrow K_{i} K_{i} \varphi$

$\neg K_{i} \varphi \rightarrow K_{i} \neg K_{i} \varphi$

$E_{i} \varphi \leftrightarrow\left(A_{i} \varphi \wedge K_{i} \varphi\right)$

$[\varphi] p \leftrightarrow(\varphi \rightarrow p)$

$[\varphi] \neg \psi \leftrightarrow(\varphi \rightarrow \neg[\varphi] \psi)$

$[\varphi](\psi \wedge \chi) \leftrightarrow([\varphi] \psi \wedge[\varphi] \chi)$

$[\varphi] K_{i} \psi \leftrightarrow\left(\varphi \rightarrow K_{i}[\varphi] \psi\right)$

$[\varphi] A_{i} \psi \leftrightarrow\left(\varphi \rightarrow A_{i} \psi\right)$

$[\varphi][\psi] \chi \leftrightarrow[\varphi \wedge[\varphi] \psi] \chi$

The inference rules:

from $\varphi \rightarrow \psi$ and $\varphi$ infer $\psi$

modus ponens

if $\vdash \varphi$, then $\vdash K_{i} \varphi$

Gödel's rule for $K_{i}$

if $\vdash \varphi$, then $\vdash[\psi] \varphi$, for any $\psi \in \Gamma_{\mathcal{L}_{D E L-A}}$

Gödel's rule for [ ] 
Let us note that the axioms for the dynamic operator extending the axioms of the S5-A logic to the axioms of the DEL-A logic enable us to eliminate announcements, one by one, from a formula of the language $\mathcal{L}_{D E L-A}$, by giving a logically equivalent formula without announcements.

To prove the soundness and completeness theorems for the logic DEL-A, we shall define a translation function $t: \Gamma_{\mathcal{L}_{D E L-A}} \mapsto \Gamma_{\mathcal{L}_{M E L-A}}$ which will enable us to translate any formula of the language of dynamic epistemic logic with the awareness operator into a formula of static epistemic logic with such an operator.

Definition 13. The translation function $t: \Gamma_{\mathcal{L}_{D E L-A}} \mapsto \Gamma_{\mathcal{L}_{M E L-A}}$ is defined as follows:

$$
\begin{aligned}
& t(p)=p, \\
& t(\neg \varphi)=\neg t(\varphi), \\
& t(\varphi \wedge \psi)=t(\varphi) \wedge t(\psi), \\
& t\left(K_{i} \varphi\right)=K_{i} t(\varphi), \\
& t\left(A_{i} \varphi\right)=A_{i} t(\varphi), \\
& t([\varphi] p)=t(\varphi \rightarrow p), \\
& t([\varphi] \neg \psi)=t(\varphi \rightarrow \neg[\varphi] \psi), \\
& t([\varphi](\psi \wedge \chi))=t([\varphi] \psi \wedge[\varphi] \chi), \\
& t\left([\varphi] K_{i} \psi\right)=t\left(\varphi \rightarrow K_{i}[\varphi] \psi\right), \\
& t\left([\varphi] A_{i} \psi\right)=t\left(\varphi \rightarrow A_{i} \psi\right), \\
& t([\varphi][\psi] \chi)=t([\varphi \wedge[\varphi] \psi] \chi) .
\end{aligned}
$$

In the next step, we shall define a measure of complexity of formulas of the language $\mathcal{L}_{D E L-A}$, that is, the function that assigns a natural number to each formula of that language.

Definition 14. The complexity measure $m: \Gamma_{\mathcal{L}_{D E L-A}} \mapsto \mathbb{N}$ is defined in the following way:

$$
\begin{aligned}
& m(p)=1 \\
& m(\neg \varphi)=1+m(\varphi) \\
& m(\varphi \wedge \psi)=1+\max (m(\varphi), m(\psi)) \\
& m\left(K_{i} \varphi\right)=1+m(\varphi) \\
& m\left(A_{i} \varphi\right)=1+m(\varphi) \\
& m([\varphi] \psi)=(4+m(\varphi)) \cdot m(\psi)
\end{aligned}
$$


The choice of such values for the complexity measure of formulas of the language $\mathcal{L}_{D E L-A}$ seems arbitrary, but it allows us to prove the following lemma:

Lemma 1. For all $\varphi, \psi, \chi \in \Gamma_{\mathcal{L}_{D E L-A}}$ :

(i) $m(\psi) \geq m(\varphi)$, if $\varphi$ is a sub-formula of $\psi$,

(ii) $m([\varphi] p)>m(\varphi \rightarrow p)$,

(iii) $m([\varphi] \neg \psi)>m(\varphi \rightarrow \neg[\varphi] \psi)$,

(iv) $m([\varphi](\psi \wedge \chi))>m([\varphi] \psi \wedge[\varphi] \chi)$,

(v) $m\left([\varphi] K_{i} \psi\right)>m\left(\varphi \rightarrow K_{i}[\varphi] \psi\right)$,

(vi) $m\left([\varphi] A_{i} \psi\right)>m\left(\varphi \rightarrow A_{i} \psi\right)$,

(vii) $m([\varphi][\psi] \chi)>m([\varphi \wedge[\varphi] \psi] \chi)$.

Proof: By induction on the complexity of a formula.

(i) Proof by induction on $\psi$. In the base step, it is enough to note that if $\psi$ is a sentential variable, then $m(\psi)=1$. In this case $m(\psi) \geq m(\psi)$ holds. Let us assume the following inductive hypothesis: $m(\psi) \geq m(\varphi)$ if $\varphi$ is a sub-formula of $\psi$, and $m(\chi) \geq m(\varphi)$ if $\varphi$ is a sub-formula of $\chi$.

(Case of negation) Suppose that $\varphi$ is a sub-formula of $\neg \psi$. Then $\varphi=\psi$ or $\varphi$ is a sub-formula of $\psi$. In the first case, the theorem holds because $m(\varphi)=m(\psi)<m(\psi)+1=m(\neg \psi)$. In the second case, we have $m(\psi) \geq m(\varphi)$ due to the inductive hypothesis. Therefore, $m(\neg \psi)=m(\psi)+1 \geq m(\varphi)$.

(Case of conjunction) Let us assume that $\varphi$ is a sub-formula of $\psi \wedge \chi$. Then $\varphi=\psi \wedge \chi$ or $\varphi$ is a sub-formula of $\psi$ or $\chi$. In the first case, trivially $m(\psi \wedge \chi) \geq m(\varphi)$. Let us consider the second case and assume that $\varphi$ is a sub-formula of $\psi$. Then, from the inductive hypothesis, $m(\psi) \geq m(\varphi)$ and consequently $m(\psi \wedge \chi)=1+\max (m(\psi), m(\chi))>$ $m(\psi) \geq m(\varphi)$. If $\varphi$ is a sub-formula of $\chi$, then by analogy we get $m(\psi \wedge \chi) \geq m(\varphi)$

(Case of the operators $K_{i}$ and $A_{i}$ ) By analogy to the case of negation.

(Case of the dynamic operator) Let us assume that $\varphi$ is a sub-formula of $\psi[\chi]$. Then $\varphi=[\psi] \chi$ or $\varphi$ is a sub-formula of $\psi$ or $\chi$. In the first case, $m([\psi] \chi) \geq m(\varphi)$. Let us consider the second case, and assume that $\varphi$ is a sub-formula of $\psi$. Since we know that $m([\psi] \chi)=(4+$ $m(\psi)) \cdot m(\chi)$ and $m(\chi) \geq 1$, from the inductive hypothesis it follows 
that $m(\psi) \geq m(\varphi)$, therefore $m([\psi] \chi) \geq m(\varphi)$. By analogy, we get $m([\psi] \chi) \geq m(\varphi)$, when $\varphi$ is a sub-formula of $\chi$.

(ii) Since $m([\varphi] p)=(4+m(\varphi)) \cdot m(p)=4+m(\varphi)$, and $m(\varphi \rightarrow p)=$ $m(\neg(\varphi \wedge \neg p))=1+m(\varphi \wedge \neg p)=1+\max (m(\varphi), m(\neg p))=1+\max (m(\varphi), 2)$, therefore $m(\varphi \rightarrow p)=1+m(\varphi)$ or $m(\varphi \rightarrow p)=3$. In both cases $m([\varphi] p)>$ $m(\varphi \rightarrow p)$.

(iii) Since $m([\varphi] \neg \psi)=(4+m(\varphi)) \cdot m(\neg \psi)=(4+m(\varphi)) \cdot(1+m(\psi))=$ $4+m(\varphi)+4 \cdot m(\psi)+m(\varphi) \cdot m(\psi)$, and $m(\varphi \rightarrow \neg[\varphi] \psi)=m(\neg(\varphi \wedge \neg \neg[\varphi] \psi))=$ $1+m(\varphi \wedge \neg \neg[\varphi] \psi)=2+\max (m(\varphi), m(\neg \neg[\varphi] \psi))=2+\max (m(\varphi), 2+$ $((4+m(\varphi)) \cdot m(\psi)))=2+\max (m(\varphi), 2+4 \cdot m(\psi)+m(\varphi) \cdot m(\psi))$, therefore $m(\varphi \rightarrow \neg[\varphi] \psi)=2+m(\varphi)$ or $m(\varphi \rightarrow \neg[\varphi] \psi)=4+4 \cdot m(\psi)+m(\varphi) \cdot m(\psi)$. In both cases $m([\varphi] \neg \psi)>m(\varphi \rightarrow \neg[\varphi] \psi)$.

(iv) Let us assume that $m(\psi) \geq m(\chi)$. Since $m([\varphi](\psi \wedge \chi))=(4+$ $m(\varphi)) \cdot m(\psi \wedge \chi)=(4+m(\varphi)) \cdot(1+\max (m(\psi), m(\chi)))=(4+m(\varphi))$. $(1+m(\psi))=4+m(\varphi)+4 \cdot m(\psi)+m(\varphi) \cdot m(\psi)$, and $m([\varphi] \psi \wedge[\varphi] \chi)=$ $1+\max (m([\varphi] \psi), m([\varphi] \chi))=1+\max ((4+m(\varphi)) \cdot m(\psi),(4+m(\varphi))$. $m(\chi))=1+((4+m(\varphi)) \cdot m(\psi))=1+4 \cdot m(\psi)+m(\varphi) \cdot m(\psi)$, therefore $m([\varphi](\psi \wedge \chi))>m([\varphi] \psi \wedge[\varphi] \chi)$. The case where $m(\chi) \geq m(\psi)$ is analogous.

(v) and (vi) are proved in an analogous way to the proof of (iii).

(vii) Since $m([\varphi][\psi] \chi)=(4+m(\varphi)) \cdot m([\psi] \chi)=(4+m(\varphi)) \cdot((4+m(\psi))$. $m(\chi))=((4+m(\varphi)) \cdot(4+m(\psi))) \cdot m(\chi)=(16+4 \cdot m(\varphi)+4 \cdot m(\psi)+m(\varphi)$. $m(\psi)) \cdot m(\chi)$, and $m([\varphi \wedge[\varphi] \psi] \chi)=(4+m(\varphi \wedge[\varphi] \psi)) \cdot m(\chi)=(4+(1+$ $\max (m(\varphi),(4+m(\varphi)) \cdot m(\psi)))) \cdot m(\chi)=(5+((4+m(\varphi)) \cdot m(\psi))) \cdot m(\chi)=$ $(5+4 \cdot m(\psi)+m(\varphi) \cdot m(\psi)) \cdot m(\chi)$, therefore $m([\varphi][\psi] \chi)>m([\varphi \wedge[\varphi] \psi] \chi)$.

Making use of Lemma 1, we can prove that each formula of $\mathcal{L}_{D E L-A}$ is equivalent to its translation in the logic DEL-A.

LEMma 2. For any formula $\varphi \in \Gamma_{\mathcal{L}_{D E L-A}}$ it holds that $\vdash_{\text {DEL-A }} \varphi \leftrightarrow t(\varphi)$.

ProOF: We conduct a proof by induction on $m(\varphi)$. In the base case, i.e. when $\varphi$ is a sentential variable, according to Definition $13, t(p)=p$ and since by Definition 12 all instances of propositional tautologies are theorems of DEL-A, we obtain $\vdash_{\text {DEL-A }} p \leftrightarrow p$. Let us assume the following inductive hypothesis: for any formula $\psi$ such that $m(\psi) \leq n$, it holds that $\vdash_{\text {DEL-A }} \psi \leftrightarrow t(\psi)$. 
(Case of negation) Let us assume that $\varphi$ is a formula of the form $\neg \psi$ such that $m(\neg \psi)=n+1$. Then $m(\psi)=n$. Therefore, by the inductive hypothesis, $\vdash_{\text {DEL-A }} \psi \leftrightarrow t(\psi)$. Hence, $\vdash_{\text {DEL-A }} \neg \psi \leftrightarrow \neg t(\psi)$. Finally, according to Definition $13, \neg t(\psi)=t(\neg \psi)$, so $\vdash_{\text {DEL-A }} \neg \psi \leftrightarrow t(\neg \psi)$.

(Case of conjunction) Let us assume that $\varphi$ is a formula of the form $\psi \wedge \chi$, such that $m(\psi \wedge \chi)=n+1$. Referring to Lemma 1(i) and Definition 14 , we get $m(\psi) \leq n$ and $m(\chi) \leq n$. Therefore, by virtue of the inductive hypothesis, $\vdash_{\text {DEL-A }} \psi \leftrightarrow t(\psi)$ and $\vdash_{\text {DEL-A }} \chi \leftrightarrow t(\chi)$. Therefore, $\vdash_{\text {DEL-A }}(\psi \wedge \chi) \leftrightarrow(t(\psi) \wedge t(\chi))$. Finally, by Definition 13, $\vdash_{\text {DEL-A }}(\psi \wedge \chi) \leftrightarrow t(\psi \wedge \chi)$.

(Case of the operators $K_{i}$ and $A_{i}$ ) By analogy to the case of negation.

(Case of the dynamic operator) Let us assume that $\varphi$ is a formula of the form $[\psi] p$, such that $m([\psi] p)=n+1$. By virtue of Lemma 1(ii), $m([\psi] p)>m(\psi \rightarrow p)$. Hence, $m(\psi \rightarrow p) \leq n$, and by virtue of the inductive hypothesis, $\vdash_{\text {DEL-A }}(\psi \rightarrow p) \leftrightarrow t(\psi \rightarrow p)$. It follows from the DEL-A axioms that $\vdash_{\text {DEL-A }}[\psi] p \leftrightarrow(\psi \rightarrow p)$. Therefore, $\vdash_{\text {DEL-A }}[\psi] p \leftrightarrow t(\psi \rightarrow p)$, and according to Definition $13, \vdash_{\text {DEL-A }}$ $[\psi] p \leftrightarrow t([\psi] p)$. The cases where $\varphi$ takes the form of $[\psi] \neg \chi,[\psi] \chi \wedge \xi$, $[\psi] K_{i} \chi,[\psi] A_{i} \chi$ and $[\psi][\chi] \xi$, are proved in an analogous way, referring to the corresponding points of Lemma 1, the inductive hypothesis, the axioms of the DEL-A logic and Definition 13.

Our Lemma 2 is crucial for proving the completeness theorem for the DEL-A logic.

TheOREM 3. Logic DEL-A is sound and complete with respect to the class of all equivalence epistemic models with the awareness function, i.e. any formula $\varphi \in \Gamma_{\mathcal{L}_{D E L-A}}$ is a DEL-A theorem iff $\varphi$ is true in all epistemic models with the awareness function, where the accessibility relations are equivalence relations.

ProOF: $(\rightarrow)$ The soundness follows from the axioms which are shown to be valid and the rules of inference which are validity-preserving. The validity of the axioms without the dynamic operator is guaranteed by the soundness of the logic S5-A. As an illustration of the validity of the remaining axioms we shall prove that $\mid={ }_{\text {DEL-A }}[\varphi] A_{i} \psi \leftrightarrow\left(\varphi \rightarrow A_{i} \psi\right)$.

Let $\mathcal{M}=\left(W,\left\{R_{i}: i \in A g\right\},\left\{\mathcal{A}_{i}: i \in A g\right\}, v\right)$ be a model of the logic DEL-A and let $s \in W$ be such that $\mathcal{M}, s \models[\varphi] A_{i} \psi$. We shall show that $\mathcal{M}, s \models \varphi \rightarrow A_{i} \psi$. Let us assume that $\mathcal{M}, s \models \varphi$. Then, according to Definition 11, we have $\mathcal{M}|\varphi, s|=A_{i} \psi$, which implies $\mathcal{M}, s \models A_{i} \psi$. 
Let $\mathcal{M}=\left(W,\left\{R_{i}: i \in A g\right\},\left\{\mathcal{A}_{i}: i \in A g\right\}, v\right)$ be a model of the logic DEL-A and let $s \in W$ be such that $\mathcal{M}, s \models \varphi \rightarrow A_{i} \psi$. We shall show that $\mathcal{M}, s \models[\varphi] A_{i} \psi$. Let us assume that $\mathcal{M}, s \models \varphi$, which implies that $\mathcal{M}, s \models A_{i} \psi$. Since $\mathcal{M}, s \models \varphi$, then $s \in \mathcal{M} \mid \varphi$. Since $s \in \mathcal{M} \mid \varphi$ and $\mathcal{M}, s \models A_{i} \psi$, then finally $\mathcal{M} \mid \varphi, s \models A_{i} \psi$. This proves that $\models_{\text {DEL-A }}$ $[\varphi] A_{i} \psi \leftrightarrow\left(\varphi \rightarrow A_{i} \psi\right)$.

$(\leftarrow)$ In order to prove completeness, let us assume that $\varphi$ is DEL-Avalid, i.e. $\models_{\text {DEL-A }} \varphi$. According to Lemma $2, \vdash_{\text {DEL-A }} \varphi \leftrightarrow t(\varphi)$. Hence, from the soundness, $==_{\text {DEL-A }} \varphi \leftrightarrow t(\varphi)$. Therefore, if $=_{\text {DEL-A }} \varphi$, then $\models_{\text {DEL-A }} t(\varphi)$. Since $t(\varphi)$ does not contain the dynamic operator, we have $\models_{\text {S5-A }} t(\varphi)$, and according to Theorem $2, \vdash_{\mathrm{S} 5-\mathrm{A}} t(\varphi)$. This implies that $\vdash_{\text {DEL-A }} t(\varphi)$, because the axioms of the logic DEL-A contain the axioms of S5-A. Hence, if $\vdash_{\text {DEL-A }} t(\varphi)$ and $\vdash_{\text {DEL-A }} \varphi \leftrightarrow t(\varphi)$, then $\vdash_{\text {DEL-A }} \varphi$.

\section{Summary}

The problem of logical omniscience is a drawback not only of static epistemic logics, but also of dynamic epistemic logics. If those systems of logic are considered to model the concept of potential knowledge represented by the operator $K_{i}$ for any agent $i$, then modeling actual knowledge remains an open question. This is an interesting problem, since the possible worlds semantics account has proven to be a highly successful framework for modeling not only epistemic notions such as knowledge, belief and information, but also the act of epistemic change. Since the possible worlds semantics framework has been widely adopted not only by philosophers, but also by computer and cognitive scientists, linguists, and artificial intelligence researchers, it is desirable to establish a dynamic model theory of knowledge that solves the problem of logical omniscience. In this article we showed that it is possible to construct a system of dynamic epistemic logic for actual knowledge of non-omniscient agents. We presented a proof system and a natural semantics for such a logic, and finally we proved the soundness and completeness theorem. In our system, actual knowledge will have additional properties once we apply some further restrictions on the awareness function, so it may be seen as a general framework of representing the actual knowledge change for different, more or less logically competent, agents. An open problem is extending the logic DEL-A in such a way that enables us to model the notion of common knowledge. 


\section{References}

[1] J. van Benthem, Epistemic Logic and Epistemology: The State of Their Affairs, Philosophical Studies, Vol. 128 (2006), pp. 49-76.

[2] R. Fagin, J. Y. Halpern, Belief, Awareness, and Limited Reasoning, Artificial Intelligence, Vol. 34 (1988), pp. 39-76.

[3] R. Fagin, J. Y. Halpern, Y. Moses, M. Vardi, Reasoning about Knowledge, MIT Press, Cambridge (1995).

[4] J. Gerbrandy, W. Groeneveld, Reasoning About Information Change, Journal of Logic, Language, and Information, Vol. 6 (1997), pp. 147-169.

[5] J. Hintikka, Knowledge and Belief, Cornell University Press, Ithaca (1962).

[6] S. Kripke, Semantical Analysis of Modal Logic (abstract), Journal of Symbolic Logic, Vol. 24 (1959), pp. 323-324.

[7] S. Kripke, Semantical Analysis of Modal Logic I. Normal Modal Propositional Calculi, Zeitschrift für Mathematische Logik und Grundlagen der Mathematik, Vol. 9 (1963), pp. 67-96.

[8] H. J. Levesque, A Logic of Implicit and Explicit Belief, Proceedings of AAAI-84, Austin (1984), pp. 198-202.

[9] J. E. Littlewood, A Mathematician's Miscellany, Meuthen, London (1953).

[10] J. Łoś, Logiki wielowartościowe a formalizacja funkcji intensjonalnych, Kwartalnik Filozoficzny, Vol. 17 (1948), pp. 57-78.

[11] A. Pap, Belief and Propositions, Philosophy of Science, Vol. 24 (1957), pp. 123-136.

[12] J. Plaza, Logics of Public Communications, [in:] M. Emrich, M. Pfeifer, M. Hadzikadic, Z. Ras (eds.), Proceedings of the 4th International Symposium on Methodologies for Intelligent Systems: Poster Session Program, Oak Ridge National Laboratory, Oak Ridge (1989), pp. 201-216.

[13] M. S. Rasmussen, Dynamic Epistemic Logic and Logical Omniscience, Logic and Logical Philosophy, Vol. 24 (2015), pp. 377-399.

[14] G. Sillari, Models of awareness, [in:] G. Bonanno, W. van der Hoek, M. Wooldridge (eds.), Logic and the Foundations of Game and Decision Theory. Vol. 2: Texts in Logic and Games, Amsterdam University Press, Amsterdam (2008), pp. 209-240.

[15] K. M. Sim, Epistemic Logic and Logical Omniscience: A Survey, International Journal of Intelligent Systems, Vol. 12 (1997), pp. 57-81. 
University of Warsaw

Institute of Philosophy

Department of Logic

ul. Krakowskie Przedmieście 3

00-927 Warszawa, Poland

e-mail: arkadiuszwojcik@uw.edu.pl 